\title{
Correlación entre el índice de masa corporal y las circunferencias corporales de niños de 4 a 10 años
}

\section{Correlation between body mass index and body circumference in children 4 to 10 year-old}

\author{
Marco Antonio Cossio-Bolaños ${ }^{1 a, 2}$, Miguel Arruda ${ }^{1 b}$, Ademir De Marco ${ }^{3 b}$ \\ ${ }^{1}$ Instituto del Deporte Universitario, Universidad Nacional de San Agustín. Arequipa, Perú. \\ ${ }^{2}$ Departamento de Ciência do desporto, Faculdade de Educação física, Universidade Estadual de Campinas. São Paulo, Brasil. \\ ${ }^{3}$ Departamento de Educação Motora, Faculdade de Educação física, Universidade Estadual de Campinas. São Paulo, Brasil. \\ a Licenciado; ${ }^{b}$ Doctor
}

\begin{abstract}
Resumen
Objetivos: Correlacionar el índice de masa corporal (IMC) con las circunferencias corporales de niños brasileros de 4 a 10 años. Diseño: Estudio de tipo descriptivo correlacional. Institución: Universidad Estatal de Campinas, Sao Paulo, Brasil. Participantes: Niños de 4 a 10 años de edad. Intervenciones: Se seleccionó 517 niños de ambos sexos de manera probabilística (estratificada), siendo 256 niños y 261 niñas. Se evaluó las medidas antropométricas de peso corporal $(\mathrm{kg})$, estatura $(\mathrm{cm})$, IMC [IMC=peso $\left(\mathrm{kg}\right.$ /estatura $\left.(\mathrm{m})^{2}\right]$ y las circunferencias corporales del brazo relajado, muslo y pantorrilla media $(\mathrm{cm})$. Para el análisis de los datos se utilizó la estadística descriptiva de media aritmética $(X)$, desviación estándar (DE) y el coeficiente de correlación producto-momento de Pearson ( $r$ ), así como para inferir el análisis de regresión múltiple stepwise paso a paso. Principales medidas de resultados: Correlación del índice de masa corporal con las circunferencias corporales. Resultados: El IMC muestra elevados coeficientes de correlación (r) con las tres circunferencias corporales. Sin embargo, cuando se aplicó la regresión estadística simple, los mejores valores fueron observados a partir de la circunferencia del brazo relajado $\left(R^{2}=0,74\right)$ para niñas y la pantorrilla media para niños $\left(R^{2}=0,70\right)$. A su vez, cuando se utilizó la regresión múltiple (brazo y pantorrilla) el nivel de explicación para las niñas aumentó a $\left(R^{2}=0,80\right)$ y para los niños a $\left(R^{2}=0,75\right)$, respectivamente. Conclusiones: Las circunferencias corporales del brazo y la pantorrilla en forma individual y conjunta podrían ser utilizadas como variables independientes del IMC, puesto que este índice por sí solo no podría distinguir el sobrepeso y exceso de grasa en niños de ambos géneros de 4 a 10 años, sobre todo cuando se encuentran en fase de crecimiento y desarrollo somático.

Palabras clave: Índice de masa corporal; pesos y medidas corporales; niños.
\end{abstract}

\begin{abstract}
Objectives: To compare body mass index (BMI) with body circumferences in 4 to 10 year-old Brazilian children. Design: Descriptive correlational type study. Setting: Universidad Estatal de Campinas, Sao Paulo, Brazil. Participants: Children 4 to 10 year-old. Interventions: We selected in a probabilistic (stratified) manner a total of 517 children both sexes, 256 boys and 261 girls. We evaluated anthropometric measurements of body weight $(\mathrm{kg})$, height $(\mathrm{cm})$, BMI $[\mathrm{BMI}=$ weight $(\mathrm{kg}) /$ height $\left.(\mathrm{m})^{2}\right]$ and relaxed mean arm girth, thigh and calf $(\mathrm{cm})$. For data analysis descriptive statistics were used for arithmetic mean (X), standard deviation (SD) and Pearson's coefficient correlation product moment $(r)$ as well as to infer step by step the stepwise multiple regression analysis. Main outcome measures: Correlation of body mass index with body circumferences. Results: BMI had high correlation coefficients $(r)$ with the three body circumferences. However, when simple statistical regression was applied, the best values were observed with the relaxed arm circumference $\left(R^{2}=0.74\right)$ for girls and children half calf $\left(R^{2}=0.70\right)$. In turn, when multiple regression was used (arm and calf) the level of explanation for girls increased to $\left(R^{2}=0.80\right)$ and children $\left(R^{2}=0.75\right)$, respectively. Conclusions: Arm circumferences and calf individually and together could be used as independent variables of BMI, since this index alone can not distinguish between overweight and excess fat in both gender children 4-10 year-old, especially when they are in the process of growth and somatic development.
\end{abstract}

Key words: Body mass index; body weights and measures; children.

\section{INTRODUCCIÓN}

El índice de masa corporal (IMC) es uno de los métodos más difundidos y utilizados para la determinación de la obesidad en adultos [IMC=peso $(\mathrm{kg})$ /estatura $\left.(\mathrm{m})^{2}\right]$ ${ }^{(1-3)}$. Este indicador ha sido muy criticado, ya que no distingue si el exceso de masa corporal es debido a la cantidad de grasa corporal, masa muscular o masa ósea ${ }^{(4)}$. A su vez, para su diagnóstico no requiere de tablas de referencia, puesto que existen valores fijos para cualquier edad, considerando que durante el transcurso de la vida es recomendable mantener constante el peso corporal ${ }^{(5)}$. Sin embargo, durante la etapa de crecimiento físico, los valores del IMC en niños y adolescentes se modifican, invalidando ese valor fijo ${ }^{(6)}$. Puesto que como índice de obesidad no es directamente aplicable a poblaciones humanas que tengan diferencias notables de altura, es decir, no es aplicable a niños y adolescentes, cuanto más alto sea un individuo, un mismo porcentaje de grasa corporal dará lugar a valores de IMC más altos ${ }^{(7)}$. Por ello, es necesario utilizar junto al IMC algunas variables de crecimiento físico, como los pliegues cutáneos ${ }^{(8)}$, diámetros, circunferencias corporales, entre otras variables, con el propósito de mejorar el diagnóstico.

En ese sentido, cabe resaltar que las herramientas más utilizadas en la evaluación de la salud pública y clínica están sustentadas en las medidas antropométricas, tales como índices corporales basados en el peso y estatura e inclusive el IMC ${ }^{(8,9)}$, en razón de su costo operacional bajo y facilidad de uso ${ }^{(10,11)}$. De esa forma, para obtener una determinación razonable del sobrepeso y distribución grasa corporal se ha propuesto varios parámetros antropométricos basados en mediciones de pliegues cutáneos y de los perímetros de diferentes segmentos corporales ${ }^{(12)}$. Se considera que la circunferencia de la cintura es un buen indicador de obesidad ${ }^{(13-}$ ${ }^{15)} \mathrm{y}$ un excelente predictor de factores de riesgo cardiovascular, superior inclusive que el IMC ${ }^{(16)}$. Por lo tanto, el objetivo del presente estudio fue correlacionar el IMC con circunferencias corporales de niños brasileros de 4 a 10 años.

\section{MÉTODOS}

Para el presente estudio se seleccionó 517 niños de ambos sexos de manera probabi- 
lística (estratificado), siendo 256 niños y 261 niñas, del Programa de interacción y desarrollo del niño y del adolescente de la Universidad Estatal de Campinas (Sao Paulo-Brasil). Todos los sujetos investigados clínicamente se encontraban sanos hasta la fecha de la evaluación.

Los padres y tutores de los niños firmaron una ficha de consentimiento, autorizando la evaluación de las medidas antropométricas, así como el proyecto contó con la aprobación del respectivo Comité Institucional de Ética en investigación.

Se utilizó los protocolos estandarizados a nivel internacional, con el propósito de ofrecer mayor grado de confiabilidad para las variables antropométricas, obteniéndose valores de error técnico de medida (ETM) inferiores al 3\% en todas las variables antropométricas. Todas las variables fueron evaluadas en horario diurno $(9,00-10,00 \mathrm{hrs})$ y realizadas por un mismo evaluador.

Los procedimientos para la antropometría fueron los siguientes:

- Masa corporal (kg): El objetivo fue determinar la masa corporal total y se utilizó una balanza digital con una precisión de $(200 \mathrm{~g})$, con una escala de 0 a $150 \mathrm{~kg}$, siguiendo las recomendaciones de Gordon, Chumlea y Roche ${ }^{(17)}$ para niños de 5 a 10 años y de 10 a 15 años.

- Estatura (cm): El objetivo fue determinar la estatura del individuo en posición ortostática, evaluándose mediante un estadiómetro de aluminio graduado en milímetros, presentando una escala de ( 0 a 2,50 m), siguiendo los procedimientos de Gordon, Chumlea, Roche ${ }^{(17)}$ y Jordan ${ }^{(18)}$.

- Circunferencias (cm): El objetivo fue evaluar la circunferencia del brazo derecho relajado, la circunferencia del muslo medio y la pantorilla media, siguiendo los procedimientos propuestos por Callaway y col. ${ }^{(19)}$, utilizando una cinta métrica de nailon milimetrada con una precisión de $0,1 \mathrm{~cm}$.

- Índice de masa corporal: El objetivo fue relacionar el peso con la estatura, a partir de la fórmula propuesta por Quetelet [IMC=peso $(\mathrm{kg}) /$ estatura $\left.(\mathrm{m})^{2}\right]^{(20)}$.
Para el presente estudio se utilizó la estadística descriptiva de media aritmética y desviación estándar, así como también el coeficiente de correlación producto momento (Pearson) para correlacionar las variables antropométricas $(p<0,001)$.

Por otro lado, respecto a la inferencia estadística, se usó el análisis de regresión múltiple stepwise paso a paso con el objetivo de determinar el porcentaje de explicación del IMC a partir de las circunferencias corporales $(p<=0,001)$. Finalmente, todo el tratamiento estadístico fue procesado en el software Sigma Estat 11.0.

\section{RESULTADOS}

La tabla 1 muestra las características antropométricas del grupo estudiado, siendo expresados en valores promedios (X) y desviación estándar (DE), donde ambos grupos muestran características similares en las variables antropométricas evaluadas (tabla 1).

El coeficiente de correlación realizado entre el IMC y las circunferencias corporales del brazo, muslo y pantorrilla muestran valores altos para ambos sexos, destacando que en las mujeres dichos valores oscilan entre $\mathrm{r}=0,81$ y 0,86 (tabla 2) y en los hombres entre $r=0,82$ y 0,84 (tabla 3). Estos coeficientes permitieron realizar regresiones estadísticas (lineal), proponiendo dos modelos, donde el primer modelo (tabla 4 y 5) utiliza una circunferencia como variable independiente y el segundo modelo (tabla 6 y 7 ) dos circunferencias corporales como variables independientes, respectivamente.

Las tablas 3 y 6 permiten observar los valores de regresión $\left(\mathrm{R}^{2}\right)$, a partir del cual, se infiere que la circunferencia del brazo en mujeres explica un $74 \%\left(R^{2}=0,74\right)$ los valores del IMC, mientras que en el caso de los hombres la circunferencia de la pantorrilla explica $70 \%\left(R^{2}=0,70\right)$, respectivamente. Sin embargo, cuando se adicionó una circunferencia corporal (modelo 2), los valores de $\mathrm{R}^{2}$ aumentaron significativamente para ambos sexos. En el caso de las mujeres, el nivel de explicación aumentó en $6 \%\left(R^{2}=0,80\right)$ cuando se adicionó la circunferencia de la pantorrilla. En relación a los hombres, se adicionó la circunferencia del brazo relajado, aumentando los niveles de explicación en $6 \%\left(\mathrm{R}^{2}=0,76\right)$. Por lo tanto, estos hallazgos demuestran que el IMC muestra valores de correlación altos y regresión con las circunferencias corporales del brazo y la pantorrilla y podrían ser utilizados junto al IMC con el propósito de disminuir el margen de error durante su diagnóstico, especialmente en la etapa de crecimiento y desarrollo somático.

\section{DISCUSIÓN}

El índice de masa corporal, durante la etapa de crecimiento físico y maduración, presenta cambios normales que se reflejan en incrementos de masa libre de grasa, más que masa grasa, en niños ${ }^{(21)}$. La masa muscular aumenta en función de la edad y la masa de grasa depende del estilo de vida y grado de actividad física de determinadas poblaciones. De esa forma, la Organización Mundial de la Salud ${ }^{(8)}$ recomienda su uso para identificar el exceso de peso en niños y adolescentes, así como en adultos.

En ese sentido, los resultados del presente estudio muestran que las circunferencias del brazo en mujeres $\left(R^{2}=0,74\right)$ y la circunferencia de la pantorrilla en

Tabla 1. Características antropométricas de niños brasileros de ambos sexos, de 4 a 10 años.

\begin{tabular}{|c|c|c|c|c|}
\hline \multirow[t]{2}{*}{ Variables } & \multicolumn{2}{|c|}{ Masculino } & \multicolumn{2}{|c|}{ Femenino } \\
\hline & $x$ & $\mathrm{DE}$ & $X$ & DE \\
\hline Edad, decimal & 7,36 & 2,12 & 7,52 & 2,15 \\
\hline Peso corporal $(\mathrm{kg})$ & 26,79 & 10,02 & 25,51 & 9,42 \\
\hline Estatura $(\mathrm{cm})$ & 122,10 & 13,14 & 120,40 & 13,59 \\
\hline $\operatorname{IMC}\left(\mathrm{kg} / \mathrm{m}^{2}\right)$ & 17,42 & 3,17 & 17,03 & 2,96 \\
\hline C. brazo $(\mathrm{cm})$ & 18,87 & 3,67 & 18,80 & 3,42 \\
\hline C. muslo (cm) & 34,30 & 6,14 & 34.04 & 5,79 \\
\hline C. pantorrilla (cm) & 25,70 & 3,74 & 25,16 & 3,58 \\
\hline$n$ & 256 & & 261 & \\
\hline
\end{tabular}


Tabla 2. Correlación producto-momento (Pearson), entre el IMC y circunferencias corporales de niñas de 4 a 10 años.

\begin{tabular}{lcccc}
\hline & IMC & C. brazo & C. muslo & C. pantorrilla \\
\hline IMC & -- & & & \\
C. brazo & $0,859^{* *}$ & -- & & \\
C. muslo & $0,807^{* *}$ & $0,776^{*}$ & -- & \\
C. pantorrilla & $0,839^{* *}$ & $0,879^{* *}$ & $0,866^{* *}$ & -- \\
\hline
\end{tabular}

IMC: índice de masa corporal, C: circunferencia. ${ }^{* *} p<0,001,{ }^{*} p<0,05$.

Tabla 3.- Correlación producto-momento (Pearson), entre el IMC y circunferencias corporales de niños de 4 a 10 años.

\begin{tabular}{lcccc}
\hline & IMC & C. brazo & C. muslo & C. pantorrilla \\
\hline IMC & -- & & & \\
C. brazo & $0,819^{* *}$ & -- & & \\
C. muslo & $0,818^{* *}$ & $0,784^{*}$ & -- & - \\
C. pantorrilla & $0,835^{\star *}$ & $0,811^{* *}$ & $0,826^{\star *}$ & - \\
\hline
\end{tabular}

IMC: índice de masa corporal, C: circunferencia. ${ }^{* *} p<0,001,{ }^{*} p<0,05$.

Tabla 4. Valores de regresión (stepwise) del IMC a partir de una circunferencia corporal en niñas de 4 a 10 años (Modelo I).

\begin{tabular}{lcccccc}
\hline & $\beta$ & Constante & $R$ & $R^{2}$ & $E E M$ & $p$ \\
\hline C. brazo & 0,744 & 3,05 & 0,859 & 0,737 & 1,5201 & $<0,001$ \\
C. muslo & 0,410 & 3,06 & 0,807 & 0,650 & 1,7445 & $<0,001$ \\
C. pantorrilla & 0,695 & $-0,447$ & 0,839 & 0,702 & 1,6203 & $<0,001$ \\
\hline
\end{tabular}

B: Beta (valores no estandarizados), R: Correlación entre la variable dependiente e independiente, $R^{2}$ : Porcentaje de explicación de la varianza (ajustado para la muestra), EEM: Error estándar de medida.

Tabla 5. Valores de predicción (stepwise) del IMC a partir de una circunferencia corporal en niños de 4 a 10 años (Modelo I).

\begin{tabular}{lcccccc}
\hline & $\beta$ & Constante & $\mathrm{R}$ & $\mathrm{R}^{2}$ & $\mathrm{EEM}$ & $p$ \\
\hline C. brazo & 0,709 & 4,06 & 0,819 & 0,670 & 1,8205 & $<0,001$ \\
C. muslo & 0,423 & 2,94 & 0,818 & 0,668 & 1,8274 & $<0,001$ \\
C. pantorrilla & 0,709 & $-0,768$ & 0,835 & 0,696 & 1,7470 & $<0,001$ \\
\hline
\end{tabular}

B: Beta (valores no estandarizados), $R$ : Correlación entre la variable dependiente e independiente, $R^{2}$ : Porcentaje de explicación de la varianza (ajustado para la muestra), EEM: Error estándar de medida.

hombres $\left(R^{2}=0,70\right)$ son las variables que explican mejor el IMC. Sin embargo, cuando se adicionó una segunda variable (circunferencia de la pantorrilla) para las mujeres, el coeficiente de determinación (regresión) aumentó a $\left(\mathrm{R}^{2}=0,80\right)$, incrementándose en $6 \%$ y, en el caso de los hombres, se adicionó la circunferencia del brazo relajado, aumentando este porcentaje en la misma proporción (6\%) $\left(\mathrm{R}^{2}=0,76\right)$, respectivamente.
En consecuencia, podemos señalar que la circunferencia, tanto del brazo como de la pantorrilla, son medidas antropométricas ampliamente utilizadas para estudios de crecimiento físico ${ }^{(22-25)}$ y en el presente estudio muestran una alta correlación con el IMC. Bayle y col. ${ }^{(26)}$ indican que el uso del IMC en forma individual es ilimitado para identificar individuos con exceso de peso corporal, donde algunos estudios reportan que el sobrepeso en niños y adolescentes no están asociados con el exceso de grasa, sino está determinado por la mayor densidad de la masa libre de grasa que la población de referencia $(27,28)$. Así también, experimentalmente se ha demostrado que el IMC no es apropiado para personas de estatura baja ${ }^{(29)}$. Esto no se puede aplicar directamente a niños y adolescentes, debido a que la altura aumenta con la edad durante el crecimiento, independientemente del contenido de grasa en el cuerpo ${ }^{(7)}$.

Por otro lado, existen pocos estudios que relacionan el IMC con variables de crecimiento físico, a excepción de la circunferencia de la cintura, que son informados por varios estudios ${ }^{(13-16)}$. En consecuencia, es necesario desarrollar investigaciones incorporando mediciones de expresión corporal y de proporcionalidad entre segmentos ${ }^{(6)}$ e inclusive de correlaciones alométricas que junto al IMC permitan un mayor grado de exactitud al momento de diagnosticar el exceso de peso y obesidad en niños y adolescentes. Esto, a pesar de que su utilización es casi universal, puesto que las bases científicas sobre las que se sustenta el concepto del IMC son poco claras ${ }^{(30)}$, dado que las leyes de la alometría establecen que la forma es modificada necesariamente por el tamaño ${ }^{(31)}$. Por lo tanto, este hecho explicaría los niveles bajos de sensibilidad, tanto en niños, adolescentes y adultos, respectivamente.

Finalmente, considerando que el sobrepeso y la obesidad necesitan de mayor precisión en la determinación de sus límites, se concluye que las circunferencias corporales del brazo y la pantorrilla podrían ser utilizadas conjuntamente con el IMC, ya que muestran altos valores de correlación y regresión en niños de 4 a 10 años en proceso de crecimiento y desarrollo.

\section{AGRADECIMIENTOS}

Se agradece a la bolsa otorgada por la CODESP de la facultad de Educación Física de la Universidad Estatal de Campinas, S.P., Brasil.

\section{REFERENCIAS BIBLIOGRÁFICAS}

1. Mei Z, Grummer-Strawn LM, Wang J, Thornton JC, Freedman DS, Pierson RN Jr, et al. Do skinfold measurements provide additional information 
Tabla 6. Valores de regresión (stepwise) del IMC a partir de tres circunferencias corporales de niñas de 4 a10 años (Modelo II).

\begin{tabular}{|c|c|c|c|c|c|c|c|c|}
\hline & \multicolumn{3}{|c|}{$\beta$} & \multirow{2}{*}{ Constante } & \multirow{2}{*}{$\mathrm{R}$} & \multirow{2}{*}{$\mathrm{R}^{2}$} & \multirow{2}{*}{ EEM } & \multirow{2}{*}{$p$} \\
\hline & C. brazo & C. muslo & C. pantorrilla & & & & & \\
\hline$A$ & 0,503 & 0,181 & -- & 1,40 & 0,886 & 0,783 & 1,3720 & $<0,001$ \\
\hline B & 0,585 & -- & 0,213 & 0,725 & 0,896 & 0,800 & 1,3270 & $<0,001$ \\
\hline C & -- & 0,177 & 0,446 & $-0,203$ & 0,854 & 0,726 & 1,5439 & $<0,001$ \\
\hline
\end{tabular}

B: Beta (valores no estandarizados), $R$ : Correlación entre la variable dependiente e independiente,

$R^{2}$ : Porcentaje de explicación de la varianza (ajustado para la muestra), EEM: Error estándar de medida.

Tabla 7. Valores de regresión (stepwise) del IMC a partir de tres circunferencias corporales de niños de 4 a10 años (Modelo II).

\begin{tabular}{|c|c|c|c|c|c|c|c|c|}
\hline & \multicolumn{3}{|c|}{ B } & \multirow{2}{*}{ Constante } & \multirow{2}{*}{$\mathrm{R}$} & \multirow{2}{*}{$\mathrm{R}^{2}$} & \multirow{2}{*}{ EEM } & \multirow{2}{*}{$p$} \\
\hline & C. brazo & C. muslo & C. pantorrilla & & & & & \\
\hline a & 0,400 & 0,235 & -- & 1,82 & 0,867 & 0,750 & 1,5865 & $<0,001$ \\
\hline b & 0,359 & -- & 0,423 & $-0,202$ & 0,870 & 0,755 & 1,5705 & $<0,001$ \\
\hline c & -- & 0,208 & 0,426 & $-0,653$ & 0,866 & 0,747 & 1,5941 & $<0,001$ \\
\hline
\end{tabular}

B: Beta (valores no estandarizados), $R$ : Correlación entre la variable dependiente e independiente, $R^{2}$ : Porcentaje de explicación de la varianza (ajustado para la muestra), EEM: Error estándar de medida.

to body mass index in the assessment of body fatness among children and adolescents? Pediatrics. 2007;119(6): e1306-13.

2. Svendsen OL, Haarbo J, Hassager C, Christiansen C. Accuracy of measurements of body composition by dual energy $\mathrm{X}$-ray absorptiometry in vivo. Am J Clin Nutr. 1993;57:605-8.

3. Brunton JA, Bayley HS, Atkinson SA. Validation and application of dual-energy $x$-ray absorptiometry to measure bone mass and body composition in small infants. Am J Clin Nutr. 1993;58:839-45.

4. Demerath EW, Schubert CM, Maynard LM, Suns $\mathrm{S}$, Chumlea WC, Pickoff A, et al. Do changes in body mass index percentile reflect changes in body composition in children? Ata from the Fels Longitudinal Study. Pediatrics. 2006;117:487-95.

5. Gibson R. Principles of nutritional assessment. New York: Oxford University Press; 1990.

6. Ortiz Hernández L. Evaluación nutricional de adolescentes. Rev med IMSS. 2002;40(2):15362.

7. Fernández López JA, Remesar X, Alemany M. Ventajas teóricas del índice de Rohrer (P/A3) sobre el índice de masa corporal (P/A2) para la estimación de la adiposidad en humanos. Rev Esp Obes. 2005;3(1):47-55.

8. World Health Organization. Physical Status: The use and interpretation of anthropometry. Geneva, Switzerland: World Health Organization; 1995.

9. Himes JH. Anthropometric assessment of nutritional status. New York: Wiley-Liss Inc.; 1991.

10. Rezende F, Rosado L, Fransceschinni S, Rosado G, Ribeiro R, Bouzas JC. Revisão crítica dos métodos disponíveis para avaliar a composição corporal em grandes estudos populacionais e clínicos. Arch Latinoamer Nutr. 2007;57(54):327-34.

11. Goran MI, Toth MJ, Poehlman ET. Assessment of research-based body composition techniques in healthy elderly men and women using the 4-compartment model as a criterion method. Int J Obes Relat Metab Disord. 1998;22:135-42.

12. Branbilla P, Bedogoni G, Moreno LA, Goran MI, Gutin B, Fox KR, et al. Cross validation of anthropometry against magnetic resonance imaging for the assessment of visceral and subcutaneous adipose tissue in children. Int I Obes (Lond). 2006;30:23-30.

13. Cuestas E, Achaval A, Garcés N, Larraya C. Circunferencia de cintura, dislipidemia e hipertensión arterial en prepúberes de ambos sexos. An Pediatr (Barc). 2007;67(1):44-50.

14. Freedman DS, Serdula MK, Srinivasan SR, Berenson GS. Relation of circumferences and skinfold thicknesses to lipid and insulin concentrations in children and adolescents: the Bogalusa Heart Study. Am J Clin Nutr. 1999, 69(2):308-17.

15. Moreno Aznar LA, Olivera JE. Obesidad. Protocolos diagnósticos y terapéuticos en pediatría. Asociación Española de Pediatría. 2002;5:353-60.

16.Van Gaal LF, Peiffer F. New approaches for the management of patients with multiple cardiometabolic risk factors. J Endocrinol Invest. 2006;29(3 Suppl):83-9.

17. Gordon C, Chumlea W, Roche A. Stature recumbent length and weight. En: Lohman T, Roche A. Martorell R. Anthropometric standardization reference manual. Champaign, Human Kinetics, 1988:3-5.
18. Jordan RJ. Crecimiento y desarrollo del niño de Cuba. Bol Med Hosp Inf México. 1980:37.

19. Callaway CW, Chumlea CW, Bouchard C, Himes JH, Lohmant T, Pollock M, Roche A, Wilmore J. Circunferences. En: Lohman T, Roche A, Martorell R. Anthropometric standarization reference manual. Champaign, IL: Human Kinetics; 1988. p. 39-53.

20. Garrow JS, Webster J. Quetelet's index (WIH2) as a measure of fatness. Int J Obes. 1985;9(2):14753.

21. Daniels S, Khuory P, Morrison J. Utility of different measures of body fat distribution in children and adolescent. Am J Epidemiol. 2000;152(12):117984.

22. Cossio-Bolanos MA, Arruda M, Gómez RA. Crecimiento físico en niños de 6 a 12 años de media altura de Arequipa - Perú (2320msnm). RICYDE. 2009;5(14):32-44.

23. Malina RM, Bouchard C, Bar-Or C. Growth maturation and physical activity. $2^{\mathrm{a}}$ ed. Champaign, IL: Human Kinetics; 2004.

24. Ross WD, Wilson NC. A strategem for proportional growth assessment. Acta Paediatr Belg. 1974;28 suppl:169-82.

25. Guedes DP, Guedes JERP. Crescimento, composição corporal e desempenho motor de crianças. Sao Paulo: CRL Balieiro; 1997.

26. Bayle MS, Bernardo AS, Salazar PG, Requejo AG, Rubino CM, Cirujano AD. Relación entre el perfil lipídico y el índice de masa corporal. Seguimiento de los 6 a los 11 años. Estudio Rivas-Vaciamadrid. An Esp Pediatr. 2006;65(3):229-33.

27.Zavaleta AN, Malina, RM. Growth and body composition of mexico-american boys 9 through 14 years of age. Am J Phys Anthropol. 1982;57:26171.

28.Garn SM, Leonard WR, Harwthorne VM. Three limitations on the body mass index. Am J Clin Nutr. 1986;44;996-7.

29. Lara-Esqueda A, Aguilar-Salinas AC, VelázquezMonroy 0, Gomez-Perez FJ, Rosas-Peralta M, Mehta $R$, et al. The body mass index is a less-sensitive tool for detecting cases with obesity-associated co-morbidities in short stature subjects. Int J Obes. 2004;28:1443-50.

30. Prentice AM, Jebb SA. Beyond body mass index. Obes Rev. 2001;2;141-7.

31.Schmidt-Nielsen $\mathrm{K}$. Why is animal size so important?. Cambridge: Cambridge University Press; 1984.

Manuscrito recibido el 6 de abril de 2010 y aceptado para publicación el 28 de mayo de 2010.

Correspondencia:

Msc. Marco Antonio Cossio Bolaños

Av. Erico Verissimo 701

Ciudad Universitaria CEP. 13083-851

Campinas, S.P. Brasil.

Correo-e: mcossio1972@hotmail.com 